\title{
POROSIMETRY BY RANDOM NODE STRUCTURING IN VIRTUAL CONCRETE
}

\author{
Piet Stroeven $^{1}$, NGhi LB LE ${ }^{\square, 1}$, LAmbertus J Sluys ${ }^{1}$ AND HuAn He ${ }^{1,2}$ \\ ${ }^{1}$ Faculty of Civil Engineering and Geosciences, Delft University of Technology, PO Box 5048, 2600 GA \\ Delft, the Netherlands; ${ }^{2} \mathrm{GeMMe}$, Minerals Engineering-Materials-Environment, University of Liège, Sart \\ Tilman B52, 4000 Liège, Belgium \\ e-mail: L.B.N.Le@tudelft.nl; P.Stroeven@tudelft.nl; L.J.Sluys@tudelft.nl; huan.he@hotmail.com \\ (Received September 6, 2011; revised February17, 2012; accepted April 24, 2012)
}

\begin{abstract}
Two different porosimetry methods are presented in two successive papers. Inspiration for the development came from the rapidly-exploring random tree (RRT) approach used in robotics. The novel methods are applied to virtual cementitious materials produced by a modern concurrent algorithm-based discrete element modeling system, HADES. This would render possible realistically simulating all aspects of particulate matter that influence structure-sensitive features of the pore network structure in maturing concrete, namely size, shape and dispersion of the aggregate and cement particles. Pore space is a complex tortuous entity. Practical methods conventionally applied for assessment of pore size distribution may fail or present biased information. Among them, mercury intrusion porosimetry and 2D quantitative image analysis are popular. The mathematical morphology operator "opening" can be applied to sections and even provide 3D information on pore size distribution, provided isotropy is guaranteed. However, aggregate grain surfaces lead to anisotropy in porosity. The presented methods allow exploration of pore space in the virtual material, after which pore size distribution is derived from star volume measurements. In addition to size of pores their continuity is of crucial importance for durability estimation. Doublerandom multiple tree structuring (DRaMuTS), introduced earlier in this journal (Stroeven et al., 2012) and random node structuring (RaNoS) provide such information.
\end{abstract}

Keywords: DEM, pore connectivity, porosimetry, star volume, virtual concrete.

\section{INTRODUCTION}

Modern computer facilities have made studies on virtual concrete simulated by discrete element method (DEM) a liable alternative for experimental investigations. The two porosimetry methods introduced in Stroeven et al. (2012) and herein are therefore intended to be applied to virtual cement pockets between neighboring aggregate grains in concrete. They are produced in an analogue version by the dynamic concurrent algorithm-based physical DEM system HADES. This is not a unique system, of course. Since the introduction of physical methods for DEM in rock mechanics by Cundall and Strack (1979), also many systems have been developed world-wide in concrete technology; in many cases however this was accomplished for specific and thus restricted purposes. Reference can be given to Jodrey and Torey (1981), Ansell and Dickinson (1986), Mościński et al. (1989), Bentz et al. (1993), O’Connor et al. (1997); Stroeven (1999), Tsunekawa and Iwashita (2001), Puri and Uomoto (2002), Williams and Philipse (2003), Li et al. (2006) and Markauskas and Kačia- nauskas (2006). Relevant discussion on this issue can be found in Stroeven et al. (2009).

For (super) high performance concrete ((S)HPC) the cement particle packing will be dense, with values of water to cement ratio $(w / c)$ as low as 0.2 . The fresh state is thereupon hydrated, a process that does not change the position of the cement grains.

During hardening, pore space is generally accepted to gradually de-percolate. This process has been demonstrated depending on the dispersion of the cement particles and will thus be reflected in a biased way by random generator-based (RG) simulation systems popular in concrete technology. References to a selection of such RG systems can be found in Stroeven et al. (2009). For specific examples, see Diekkämper (1984), Roelfstra (1989), van Breugel (1991), Bentz et al. (1993), Meakawa et al. (1999) and Bishnoi and Scrivener (2009). Significant differences between the de-percolation process characteristics obtained by (analogue) DEM- and RG-based systems and with the digitized model of Garboczi and Bentz (2001) 
have been shown in Chen et al. (2006). For details of the hydration algorithms in the case of spherical cement grains, see Stroeven et al. (2011). This is similar to hydration algorithms developed elsewhere (Navi and Pignat, 1999; Stroeven, 1999).

Experimental approaches can provide information on porosity and pore size distribution. An obvious method is quantitative image analysis, which is also used in concrete technology. However, the most popular approach is definitely mercury intrusion porosimetry (MIP). The latter offers significantly biased information, however, because of the unrealistic schematization of the geometry of the pore channels and the neglect of the so called bottle necks in the pore system (Diamond, 2000). Insight into pore connectivity, which is of crucial importance for estimating durability risks, therefore requires other approaches.

The presented novel methods (see also Stroeven et al., 2012) allow making a distinction between continuous pores (trunks) stretching over the full dimensions of the cement pocket, isolated pores and deadend pores branching off the trunks. Particularly the latter have been demonstrated making the connections between pore trees of neighboring interfacial transition zones (ITZs), so that finite values of connected porosity were found for bulk paste. This was demonstrated by the earlier described DRaMuTS example in Stroeven et al. (2012).

Volume-based pore size distribution is derived from star volume measurements in 3D, as demonstrated also earlier. Of course, this information can be transferred to a traditional number-based pore size distribution.

\section{RANDOM NODE STRUCTURING}

\section{ALGORITHM}

Random node structuring (RaNoS) is a general approach, which can be used for porosimetry in hydrated virtual (blended) cement pastes or for other purposes, such as porosimetry in packing of grains. Fig. 1 presents a 2-D scheme for RaNoS. It starts by generating with a pseudo-random generator algorithm a system of nodes uniformly at random (UR) dispersed in container space. Then, the nodes situated in solid phase are eliminated from further consideration. This yields nodes UR dispersed inside pores as a detection system of the 3D capillary pore system in cement paste. The next step is a structuring process for the node system in which the relationships between the nodes are built up by unobstructed straight line segments connecting the nodes.

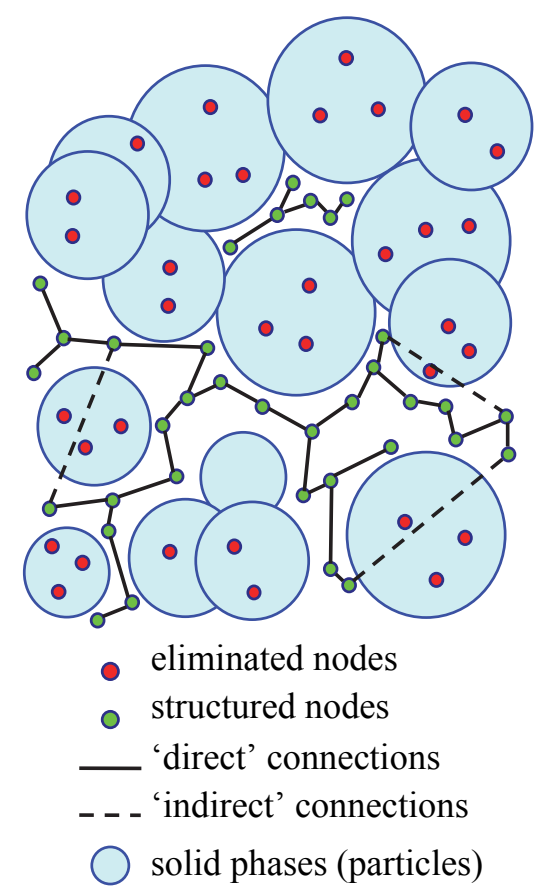

Fig. 1. Structure of random nodes in RaNoS method.

When two nodes can be connected together by a straight line that does not intersect with any part of the solid phases, such two nodes have 'direct connection'. This initiates the node-clustering process. A cluster involves all nodes that are directly connected. When two of such clusters during this process can be directly connected, they merge into a new one. Hence, once the clustering process is finished, there are a number of structured clusters of nodes. In each of these clusters, the nodes are mutually connected reflecting the connectivity among pores in the virtual cement paste. Based on the node structure, pore characteristics such as porosity, degree of percolation, pore location distribution and pore size distribution are assessed.

\section{IMPLEMENTATION}

\section{Elimination of nodes in solid phases}

As mentioned above, to obtain nodes that are uniformly at random distributed in pore space, an elimination process is required of the nodes interfering with solid phase. For particles simulated by spheres, this can easily be accomplished. For particles simulated by digital-image-based model (Bentz and Garboczi, 1991) this is also easily carried out by determining the phase of the voxel in which the considered point is situated. 
For particles simulated by polyhedrons, this is more complicated. Such particles have been employed earlier for simulating crushed rock aggregate and for cement grains (He, 2010). An efficient way to detect if a point is inside a convex polyhedron was presented in $\mathrm{He}(2010)$. If $n_{i} \bullet\left(X-V_{i}\right)<0$ is satisfied for all facets of the polyhedron, the considered point $X$ is inside. $n_{\mathrm{i}}$ is the normal vector of facet $i$ pointing outside of the polyhedron; $V_{\mathrm{i}}$ is a vertex of facet $i$. This, however, can not be used for the case of nonconvex polyhedrons.

Fig. 2 illustrates a more general way for this detection. A probing ray emanating from the considered node in a random direction is generated. In fact, this ray is a line segment (limited by two ends) and its length must be long enough to possibly go through the polygon. If the number of intersections of the ray and the polyhedron's surfaces (polygons) is odd then the point is inside the polyhedron, otherwise it is outside the polyhedron.

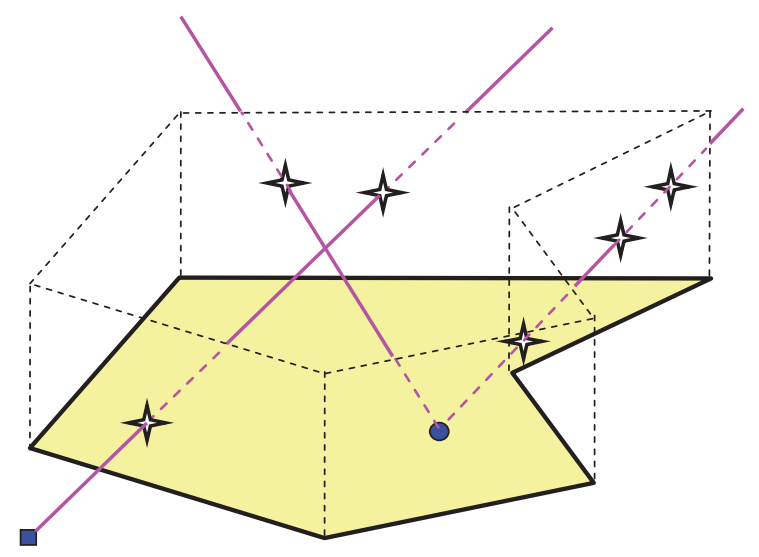

- node inside the polyhedron

- node outside the polyhedron probing ray

$\downarrow$ intersections of probe rays and the polyhedron's facets

Fig. 2. Determination whether a point is inside or outside a polyhedron.

Although the example case is a simple one, whereby spherical cement grains are used, recent synchrotron-based X-ray imaging results by Garboczi and Bullard (2004) revealed cement particles to explicitly display convex as well as non-convex parts in their surface. In our research, so far, mixtures of ellipsoidal cement particles or of polyhedrons were employed for simulating cement particle packing (He, 2010). Hereby the surface to volume ratio is similar to that found by Garboczi and Bullard (2004) in the aforementioned experiments. Since hydration is a surface-driven phenomenon this would be a next step in simulating hydrating systems among other things for porosimetry. Ultimately, also particles with non-convex surface elements should be considered, since this will influence the formation of hydration products in a densely packed binder system. For this purpose, the hydration algorithms will require significant adaptations. Yet, the HADES simulation system should be advanced enough to render possible performing such investigations in the near future.

\section{Detection of connection between two nodes}

As mentioned above, the RaNoS method involves a system of random nodes that are pair wise connected by unobstructed line segments. The line segment will be considered as being unobstructed if it does not have any intersection with neighboring particles. In case particles are simulated by spheres, the intersection detection reduces to that of a line and a sphere. In case particles are simulated by the digital-imagebased model (Bentz and Garboczi, 1991), a series of points with constant spacing is distributed along the line segment. Hence, the segment line is unobstructed if there is no point situated in any voxel representing a solid phase. In case particles are simulated by polyhedral shapes, the detection procedure is the same as earlier described. Since two ends of the line segments are outside particles, the number of intersections is only either zero or an even number. If the number is zero then there is no intersection. Otherwise, such an intersection exists.

\section{Localized and parallel computing}

The RaNoS method centers on finding direct connections between neighboring nodes. The cell method is utilized for this purpose. Each cell contains a list of particles that interfere with the cell and a list of associated nodes. The cells render possible executing localized operations to improve the speed of computation. So, the clustering process is first applied locally in each cell. Thereupon, the clustering process is applied in the whole simulated space. Moreover, since local clustering processes can be implemented independently and simultaneously, "parallel computing processing', the advanced feature of some programming languages, is also applied to speed up the computations.

\section{Detection of pore connectivity}

Considering a cubic sample pocket of cement paste, two types of capillary pores can be distinguished. The percolated pores form continuous entities connecting two opposite sides of the specimen. The 
de-percolated pores or the isolated pores might be connected together but are not connected to both sides of the pocket. The connectivity of pores is defined as the volume ratio of percolated pores to the total of capillary pores. Since the system of UR dispersed nodes can be considered as a discretized system of pore space, the pore connectivity can be estimated as the ratio of the number of nodes that are connected to the two opposite sides, to the total number of random nodes. For assessment of pore connectivity, nodes should also be uniformly at random distributed on the opposite outer surfaces of the specimen and incorporated in the clustering process. When a cluster of nodes contains at least one node in each of the two opposite surfaces, this cluster represents a percolated pore channel. In analyzing characteristics of the pore system, e.g., porosity, connectivity, pore size distribution, the nodes situated in the two end surfaces are not taken into account, however.

\section{RaNoS EXAMPLE}

Two cubic pockets of simulated cement paste are considered in this study. The characteristics of the two pockets are shown in Table $1(\mathrm{P}=$ periodic, $\mathrm{R}=$ rigid boundary). Fresh cement particles were generated and packed with the HADES system. The initial size of the pocket was eight times reduced in the dynamic packing process. A rigid surface leads to the formation of an interfacial transition zone (ITZ); an interesting feature in concrete technology. However, it is the inevitable result of the well-known wall effect, because the cement particles will pile up differently close to the wall, constituted by the aggregate grain's surfaces. In general, higher porosity and lower strength are reported as in bulk (Ollivier et al., 1995; Maso, 1996; Scrivener, 1999; Diamond and Huang, 2001; Stroeven and Stroeven, 2001). This paper does not pursue discussing the phenomenon of ITZ development in concrete, however.
Next, hydration was realized by the hybrid simulation approach described in Stroeven et al. (2011). Finally, the pore structure of the samples was investigated by $\mathrm{RaNoS}$ at ultimate degree of hydration $(\mathrm{DOH})$. Due to shortage of water for chemical reactions, the hydration process of the samples stops at ultimate $\mathrm{DOH}$ of 0.727 . Note that the maximum grain size of the simulated cement is about three times smaller than in practice. Hence, this should be accounted for in the evaluation of obtained results (such as the extension of the boundary zone).

Fig. 3 presents the random nodes distributed in pore space of the two cement pockets. Based on these systems, porosity of the $\mathrm{C} 1$ and $\mathrm{C} 2$ pockets was found to amount $5.57 \%$ and $5.59 \%$, respectively. The associated connected pore fractions of the $\mathrm{C} 1$ and $\mathrm{C} 2$ pockets were $98.53 \%$ and $97.58 \%$. The gradient structure of pore volume as a function of distance from the rigid (R) surface is displayed in Fig. 4 and compared with observations on bulk paste (container with six periodic (P) boundaries). Note the similarity with the gradient structures shown in Fig. 6 in Stroeven et al. (2012). Fig. 5 presents volume-based pore size distribution functions assessed by the star volume measurements.

Fig. 6 shows sensitivity analyses for the connected fraction of porosity of the $\mathrm{C} 1$ pocket by $\mathrm{RaNoS}$ and by the digital technique proposed by Hosen and Kapelman and cited in Navi and Pignat (1999). In order to check connectivity in the digital technique, the microstructure is subdivided into a 3D regular lattice of cubic volume-pixels (voxels), each of which represents either a solid or a pore part. Then a clustering process for pore voxels is implemented to distinguish two types of pore voxels: voxel clusters that can be connected to both top and bottom surfaces and those which are isolated. The connected fraction is then calculated as the ratio of the number of voxels belonging to the first type to that belonging to the second type.

Table 1. Characteristics of simulated cement samples.

\begin{tabular}{|c|c|c|c|c|c|c|}
\hline & $\begin{array}{l}\text { Boundary } \\
\text { conditions }\end{array}$ & $\begin{array}{l}\text { Diameter range } \\
\qquad(\mu \mathrm{m})\end{array}$ & $\begin{array}{l}\text { Specific surface area } \\
\left(\mathrm{cm}^{2} / \mathrm{g}\right)\end{array}$ & $\begin{array}{c}\text { Size of pocket } \\
(\mu \mathrm{m})\end{array}$ & $\begin{array}{l}\text { Initial } W / C \\
\quad \text { ratio }\end{array}$ & $\begin{array}{c}\text { Number of } \\
\text { particles }\end{array}$ \\
\hline $\mathrm{C} 1$ & $6 \mathrm{P}$ & \multirow{2}{*}{$1 \sim 30$} & \multirow{2}{*}{1838} & \multirow{2}{*}{100} & \multirow{2}{*}{0.304} & \multirow{2}{*}{4060} \\
\hline $\mathrm{C} 2$ & $4 P+2 R$ & & & & & \\
\hline
\end{tabular}



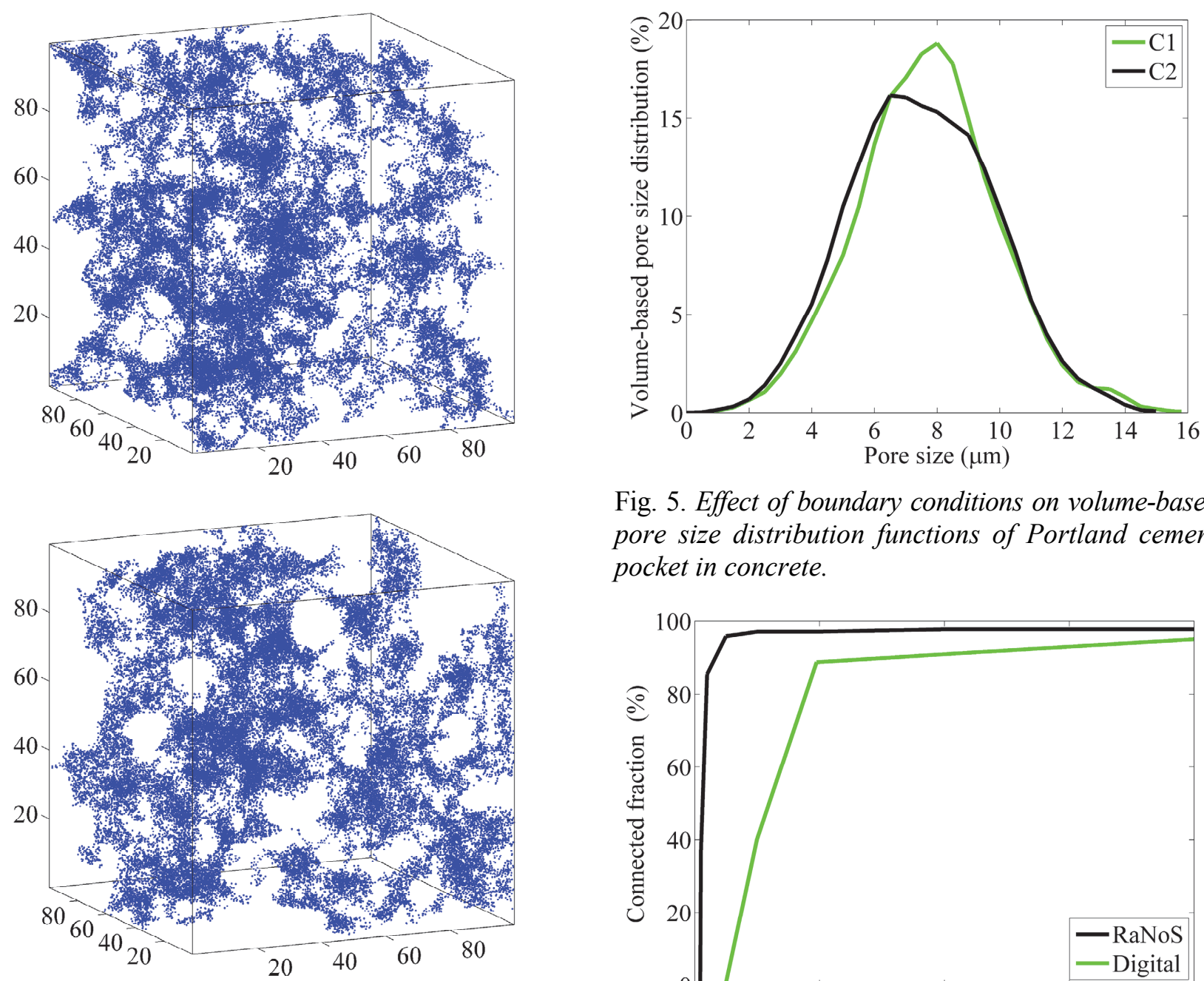

Fig. 5. Effect of boundary conditions on volume-based pore size distribution functions of Portland cement pocket in concrete.

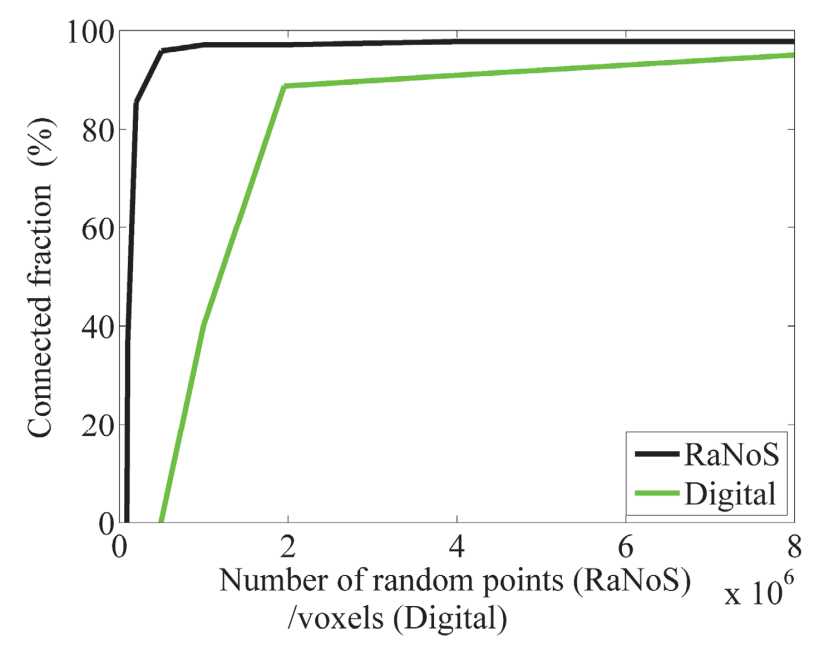

in pore space of cement pocket $C 1$ (top) and $C 2$ (bottom) (unit of axes is $\mu m$ ).

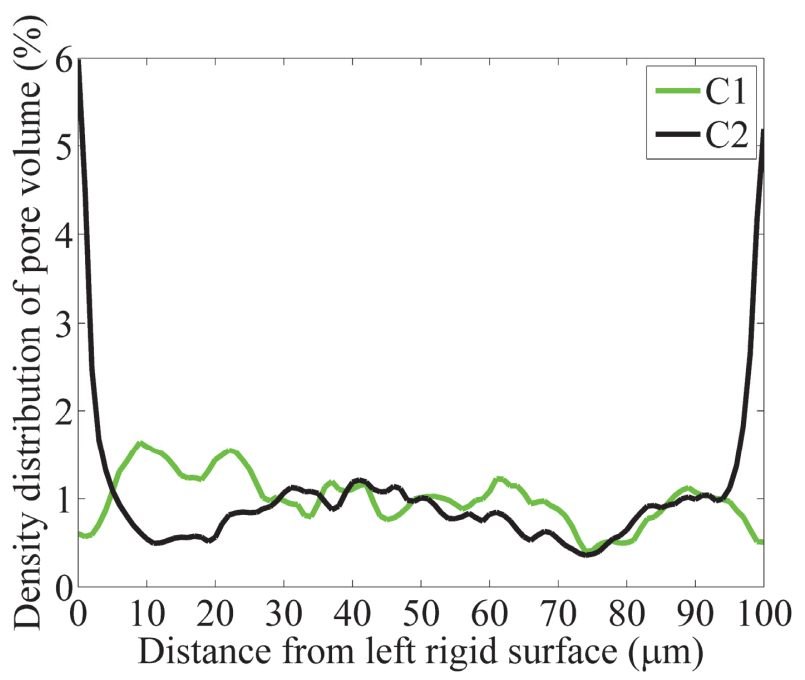

Fig. 4. Density distribution of pore volume as function of distance from the left rigid surface.

Fig. 6. Sensitivity analyses of connected fraction of porosity on a number of sampling nodes (C1 pocket) by RaNoS versus 'digital technique' (cited in Navi and Pignat, 1999).

Fig. 6 demonstrates that the connected pore fraction becomes stable (accepting only a very small change) with more than 1 million of random points in RaNoS. In the digital technique a far higher number would be required, revealing the efficiency of the new technique.

\section{DISCUSSION}

The two novel porosimetry methods introduced herein (RaNoS) and in Stroeven et al. (2012) (DRaMuTS) are somewhat similar but still have different characteristics and potentialities. In RaNoS a system of UR dispersed nodes is first generated and then a clustering process starts, ultimately resulting in groups of connected nodes. Contrary, in DRaMuTS a 
system of virtual trees consisting of vertices (nodes) and edges (line connecting a pair of nodes) are first generated in pore space. Note that the vertices are distributed randomly but not uniformly as a result of introduced improvements to speed up exploration of pore space. So, this cannot be used to analyze pore characteristics. For that purpose, a second system of UR dispersed nodes is generated. A common point of the two methods is therefore that pore characteristics (such as pore size distribution) are analyzed on the basis of a system of UR dispersed nodes. The tree systems in DraMuTS additionally render possible exploring pore space with respect to topological properties. Trunks of the trees can be defined as pore network parts connected to external sides of the pocket, whereas others parts of the trees branch off the trunks, basically form-ing dead-end pores unless coalescing with those of a neighboring tree. Finally, isolated pores can be distinguished. This topological exploration is impossible in RaNoS.

The porosimetry study by Chen et al. (2004; 2006), whereby use was made of serial sectioning and $3 \mathrm{D}$ reconstruction, led to the conclusion that (for low $w / c$-ratios) continuous porosity was zero outside a narrow zone adjacent to the aggregate surface. Hence, diffusivity of concrete would rely on a spatial pore network structure whereby a significant degree of ITZ percolation should provide for the connection in the continuous pore channels circumventing the aggregate grains. This would not be in agreement with the fore-going.

Instead, the porosimetry applications of the presented methods demonstrate that pore continuity is realized outside the aforementioned narrow zones with high degree of continuous porosity. The significant bulk value displayed in Fig. 4 is most probably due to dense network structures of (basically dead-end) pores branching off the continuous channels in the narrow zones. Even without ITZ percolation, these pore network structures can connect to form transport routes in bulk paste.

Data in Fig. 4 render possible roughly estimating in an effective medium approach the impact of higher ITZ porosity on global porosity, and thus on the expected ITZ contribution to durability issues such as chloride diffusion. Of course, ITZs partly overlap in a dense random packing of aggregate. Garboczi and Bentz (1997) analytically approached this problem for spherical aggregate grains. Zheng et al. (2011a) extended the analytical approach to RGsimulated dispersions of ellipsoidal aggregate and applied it for estimating chloride diffusivity in concrete
(Zheng et al., 2011b). Of course, further extension and generalization as to grain shape would readily be possible by HADES simulation.

As an example, for a Fuller distribution with maximum aggregate size of $16 \mathrm{~mm}$, ITZ thickness is taken as $t=30 \mu \mathrm{m}$ or $50 \mu \mathrm{m}$ (selected from literature). Zheng et al. (2011a) provide numerical data for ITZ volume fraction $\left(V_{\text {ITZ }}\right)$ at spherical aggregate volume fractions $\left(V_{\mathrm{agg}}\right)$ of respectively 0.6 and 0.75 as listed in Table 2. Fig. 4 allows approximately estimating average porosity over an ITZ scaled down by a factor of 3 .

Table 2. ITZ volume fraction for different values of aggregate volume fraction and ITZ thickness.

\begin{tabular}{ccc}
\hline & \multicolumn{3}{c}{$V_{\mathrm{ITZ}}$} \\
\hline$V_{\text {agg }}$ & 0.6 & 0.75 \\
$t=30 \mu \mathrm{m}$ & 0.045 & 0.060 \\
$t=50 \mu \mathrm{m}$ & 0.085 & 0.105 \\
\hline
\end{tabular}

For instance, with a chosen ITZ thickness of $t=30 / 3=10 \mu \mathrm{m}$ in the virtual material, a value of 1.98 is obtained for the ratio of ITZ porosity to bulk porosity, whereas with $t=50 / 3 \approx 17 \mu \mathrm{m}$ this value is 1.32. Hence, based on data in Table 2 and Fig. 4 , the global paste porosity $\bar{p}$ can be calculated for $V_{\text {agg }}=$ 0.6 :

$$
\left\{\begin{array}{l}
(t=30 \mu m) ; \bar{p}=\frac{p_{\text {bulk }} V_{\text {bulk }}+1.98 p_{\text {bulk }} V_{I T Z}}{V_{\text {paste }}} \\
=\frac{p_{\text {bulk }}(0.4-0.045)+1.98 p_{\text {bulk }} 0.045}{0.4}=1.110 p_{\text {bulk }} \\
(t=50 \mu m) ; \bar{p}=\frac{p_{\text {bulk }} V_{\text {bulk }}+1.32 p_{\text {bulk }} V_{I T Z}}{V_{\text {paste }}} \\
=\frac{p_{\text {bulk }}(0.4-0.085)+1.32 p_{\text {bulk }} 0.085}{0.4}=1.068 p_{\text {bulk }}
\end{array}\right.
$$

and for $V_{\text {agg }}=0.75$ :

$$
\left\{\begin{array}{l}
(t=30 \mu \mathrm{m}) ; \bar{p}=\frac{p_{\text {bulk }} V_{\text {bulk }}+1.98 p_{\text {bulk }} V_{I T Z}}{V_{\text {paste }}} \\
=\frac{p_{\text {bulk }}(0.25-0.06)+1.98 p_{\text {bulk }} 0.06}{0.25}=1.235 p_{\text {bulk }} \\
(t=50 \mu m) ; \bar{p}=\frac{p_{\text {bulk }} V_{\text {bulk }}+1.32 p_{\text {bulk }} V_{I T Z}}{V_{\text {paste }}} \\
=\frac{p_{\text {bulk }}(0.25-0.105)+1.32 p_{\text {bulk }} 0.105}{0.25}=1.134 p_{\text {bulk }}
\end{array}\right.
$$

in which $V$ and $p$ denote, respectively, the volume fraction and porosity. 
This demonstrates that the accuracy of estimating the thickness of the ITZ can be low. The aggregate grain surfaces increased overall porosity in the paste as a consequence by about $9 \%$ in the case of more dispersed aggregate grains and by $19 \%$ in the dense aggregate case. In this effective media approach, the porosity in the aggregate is neglected.

The presented method as well as the earlier introduced method, DraMuTS (Stroeven et al., 2012), offers new information on the pore network structure in hardening cement paste. This should have influence on models nowadays used for prediction of transport through porous concrete. Such methods were discussed in $\mathrm{Hu}$ (2004). A new approach to durability estimation might however require setting sensitivity on a somewhat lower level than in the plateau range of Fig. 6. This would modify the pore network structure in Fig. 3 into a partly de-percolated one, which is more in accordance with the commonly accepted concept in this field.

Moreover, Fig. 5 already demonstrates that introduction of ITZs leads to pore refinement. In Le and Stroeven (2012) it is shown that this phenomenon primarily takes place in the ITZ. For durability estimation in the case that mechanical influences (i.e., deformation and micro cracks developed due to loading in the ITZ) can be neglected, the effect of ITZ percolation can be expected not significant, which is in agreement with Zheng et al. (2011b).

The relative potentialities of the two methods should still be explored as to efficiency, reliability and capabilities for various applications (not necessarily limited to porosimetry in hydrated systems). At the moment we do so, but it is likely that both methods will have their own merits under different circumstances. Generally speaking, although DRaMuTS is more advanced for pore space exploration, $\mathrm{RaNoS}$ is cheaper and simpler.

The new methods concern physical DEM producing an analogue representation of concrete. Not discussed herein are stochastic DEM that have been introduced in Stroeven et al. (2009), whereby among other things reference was made to Dequiedt et al. (2001), who claim such models to be even more promising than the physical ones.

\section{CONCLUSIONS}

Novel approaches to porosimetry in virtual concrete are presented that combine reliability and economy. These methods rely on realistic analogue simulations of fresh cementitious materials by a dynamic algorithm-based DEM system, HADES. During maturation, a process of pore de-percolation takes place that can be numerically assessed. To do so, the developing pore network structure is analyzed by modern methods of which development is inspired by experiences in robotics. The first of the two presented methods (Stroeven et al., 2012) renders possible separating between continuous pores (trunks), dead-end pores branching off such pores, and isolated dead-end pores. Tree-like pore network structures are obtained of which density is highest in the ITZ. As to this aspect they confirm results obtained by the far more laborious approach of serial sectioning and 3D reconstruction.

Aggregate grains have been demonstrated coming close enough to cause neighboring ITZs to overlap or percolate (at least) partly. This is the location where probability is highest for pore trees associated with neighboring aggregate grains to connect through their branches of dead-end pores. The result is that the dense random packing of the aggregate leads to connected pathways (trunks) over the full extension of concrete specimens or elements. However, it is demonstrated by application of the developed porosimetry methods that also bulk cement paste between somewhat more remote aggregate grains contains continuous pores through mutual connection of deadend pores branching off the trunks, although density is lower that in the ITZ. As to this aspect, results deviate from those obtained by serial sectioning and $3 \mathrm{D}$ reconstruction.

A simple effective medium approach demonstrates that overall porosity is as a consequence not dominated by higher (continuous) porosity in the ITZ. Moreover, the effect of somewhat higher average ITZ porosity on global hydraulic properties can be expected even further reduced because of the relative fineness of ITZ's pore system. Hence, transport of harmful substances (including water) through concrete can be expected not significantly influenced by the degree of ITZ percolation in case mechanical influences by loading are neglected.

The complex and tortuous pores in the network structure can easily be analyzed as to size distribution by application of star volume measurements. This is a well-known experimental approach in life sciences. Its usage is shown straight forward when dealing with the 3D virtual pore structure. However, it can equally be applied to $2 \mathrm{D}$ sections (physical experiments) from which an unbiased estimate of local pore size can be obtained. Such data (in 2D and 
3D alike) obtained from a large number of random points in pore space (again, 2D and 3D alike) are readily transformed into volume-based cumulative pore size distributions and through differentiation into pore size distributions.

\section{REFERENCES}

Ansell GC, Dickinson E (1986). Sediment formation by Brownian dynamics simulation: Effect of colloidal and hydrodynamic interactions on the sediment structure. J Chem Phys 85:4079-86.

Bentz DP, Garboczi EJ (1991). Percolation of phases in a three-dimensional cement paste microstructural model. Cem Concr Res 21:325-44.

Bentz DP, Garboczi EJ, Stutzman PE (1993). Computer modelling of the interfacial transition zone in concrete. In: Maso JC, ed. Interfaces in cementitious composites. London: E\&FN Spon, p.p. 259-68.

Bishnoi S, Scrivener KL (2009). $\mu$ ic: A new platform for modelling the hydration of cements. Cem Concr Res 39:266-74.

Breugel KV (1991). Simulation of cement hydration and formation of structure in hardening cement based materials. PhD Thesis, Delft University of Technology. Delft.

Chen HS, Ye G, Stroeven P (2004). Computer simulation of structure of hydrated cement paste enclosed by interfacial transition zone in concrete. In: Setzer MJ, Palecki S, eds. Proceedings of International Conference on Durability of High Performance Concrete and Final Workshop of CONLIFE, 2004 September 23-24; Essen, Germany. Freiburg: Aedificatio Publishers, 133-44.

Chen HS, Stroeven P, Ye G, Stroeven M (2006). Influence of boundary conditions on pore percolation in model cement paste. Key Eng Mater 302-303:486-92.

Cundall PA, Strack ODL (1979). A discrete numerical model for granular assemblies. Geotech 29: 47-65.

Dequiedt A-S, Coster M, Chermant J-L, Jeulin D (2001). Towards a model of concrete mesostructure. Cem Concr Compos 23:289-97.

Diamond S (2000). Mercury porosimetry: an inappropriate method for the measurement of pore size distributions in cement-based materials. Cem Concr Res 30:1517-25.

Diamond S, Huang J (2001). The ITZ in concrete - a different view based on image analysis and SEM observations. Cem Concr Compos 23:179-88.

Diekkämper R (1984). Ein Verfahren zur numerischen Simulation des Bruch- und Verformungsverhaltens spröder Werkstoffe. Ruhr-Universität Bochum.

Garboczi EJ, Bentz DP (1997). Analytical formulas for interfacial transition zone properties. Adv Cem Based Mater 6:99-108.

Garboczi EJ, Bentz DP (2001). The effect of statistical fluctuation, finite size error, and digital resolution on the phase percolation and transport properties of the
NIST cement hydration model. Cem Concr Res 31: 1501-14.

Garboczi EJ, Bullard JW (2004). Shape analysis of a reference cement. Cem Concr Res 34:1933-7.

$\mathrm{He} \mathrm{H}$ (2010). Computational modelling of particle packing in concrete. PhD Thesis, Delft University of Technology. Delft.

Hu J (2004). Porosity in concrete - morphological study of model concrete. PhD Thesis, Delft University of Technology. Delft.

Jodrey WS, Tory EM (1981). Computer simulation of isotropic, homogeneous, dense random packing of equal spheres. Powder Technol 30:111-8.

Le NLB, Stroeven P (2012). Strength and durability evaluation by DEM approach of green concrete based on gap-graded cement blending. Adv Mater Res 450-451: 631.

Li JF, Chen CS, Yu BY, Wei WJ (2006). Simulation of colloidal particle packing for photonic bandgap crystals. J Am Ceram Soc 89:1257-65.

Maekawa K, Chaube R, Kishi T (1999). Modelling of concrete performance: hydration, microstructure formation and mass transport. London: E\&FN Spon.

Markauskas D, Kačianauskas R (2006). Compacting of particles for biaxial compression test by discrete element method. J Civ Eng Manage XII:153-61.

Maso JC (1996). Interfacial transition zone in concrete. In: RILEM report 11. E and FN Spon.

Mościński J, Bargieł M, Rycerz ZA, Jacobs PWM (1989). The force-biased algorithm for the irregular close packing of equal hard spheres. Mol Simul 3:201-12.

Navi P, Pignat C (1999). Simulation of cement hydration and the connectivity of the capillary pore space. Adv Cem Based Mater 4:58-67.

O'Connor RM, Torczynski JR, Preece DS, Klosek JT, Williams JR (1997). Discrete element modeling of sand production. Int J Rock Mech Min Sci 34:231.e1-e15.

Ollivier JP, Maso JC, Bourdette B (1995). Interfacial transition zone in concrete. Adv Cem Based Mater 2:30-8.

Puri UC, ASCE M, Uomoto T (2002). Characterization of distinct element modeling parameters for fresh concrete and its applications in shotcrete simulations. J Mater Civ Eng 14:137-44.

Roelfstra PE (1989). A numerical approach to investigate the properties of numerical concrete. $\mathrm{PhD}$ Thesis, Ecole Polytechnique Fédérale de Lausanne. Lausanne.

Scrivener KL (1999). Characterization of the ITZ and its quantification by test methods. In: Alexander MG, Arliguie G, Ballivy G, Bentur A, Marchand J, eds. Engineering and transport properties of the ITZ in cementitious composites, RILEM Report 20. Paris: RILEM Publications SARL, pp. 3-15.

Stroeven M (1999). Discrete numerical modelling of Composite Materials - application to cementitious materials. $\mathrm{PhD}$ Thesis, Delft University of Technology. Delft. 
Stroeven P, Stroeven M (2001). Reconstructions by SPACE of the Interfacial Transition Zone. Cem Concr Compos 23:189-200.

Stroeven P, Hu J, Stroeven M (2009). On the usefulness of discrete element computer modeling of particle packing for material characterization in concrete technology. Comput Concr 6:133-53.

Stroeven P, Le NLB, Stroeven M, Sluys LJ (2011). Discrete element modeling approach to porosimetry for durability risk estimation of concrete. In: Oñate E,Owen DRJ, eds. Proceedings of the II International Conference Particle-based Methods, Fundamentals and Applications (on CD), 2011 October 26-28; Barcelona, Spain.

Stroeven P, Le NLB, Sluys LJ, He H (2012). Porosimetry by double random multiple tree structuring. Image Anal Stereol 31:55-63.

Tsunekawa H, Washita K (2001). Numerical simulation of triaxial test using two and three dimensional DEM. In:
Kishino Y, eds. Proceedings of the $4^{\text {th }}$ International Conference on micromechanics of granular media, powders and grains, 2001 May 21-25; Sendai. Rotterdam: Balkema, 177-80.

Williams SR, Philipse AP (2003). Random packings of spheres and spherocylinders simulated by mechanical contraction. Phys Rev E Stat Nonlin Soft Matter Phys 67:051301/1-9.

Zheng JJ, Guo ZQ, Huang XF, Stroeven P, Sluys LJ (2011a). ITZ volume fraction in concrete with spheroidal aggregate particles and application: Part I. Numerical algorithm. Mag Concr Res 63:473-82.

Zheng JJ, Guo ZQ, Huang XF, Stroeven P, Sluys LJ (2011b). ITZ volume fraction in concrete with spheroidal aggregate particles and application: Part II. Prediction of the chloride diffusivity of concrete. Mag Concr Res 63:483-91. 